\title{
ADJACENT SEGMENT DEGENERATION AFTER DECOMPRESSION AND FUSION SURGERY FOR DEGENERATIVE LUMBAR SPINAL STENOSIS: A RETROSPECTIVE COMPARATIVE EVALUATION
}

\author{
๑ Evren KARAALI, @ FIrat SEYFETTINOǦLU
}

University of Health Sciences Turkey, Adana City Training and Research Hospital, Clinic of Orthopaedics and Traumatology, Adana, Turkey

\begin{abstract}
Objective: The information available on the association between transforaminal lumbar interbody fusion (TLIF) surgery and adjacent segment degeneration (ASD) in lumbar spinal stenosis (LSS) patients is extremely limited. To explore the risk factors involved in the development of ASD after decompression and fusion surgery for LSS.

Materials and Methods: This study subjects were patients who underwent lumbar posterior segmental instrumentation and spinal decompression surgery for degenerative LSS, in L4-5 or L5-S1, during 2010-2015. These patients were classified into two groups based on their stage of ASD development. The diagnosis of ASD was based on magnetic resonance imaging findings. The study groups were compared to determine the risk factors for ASD.

Results: A total of 162 patients (68 men, 94 women) of a mean age 60.76 5.4 years (age range: 37-89 years) were evaluated. The mean followup period for these patients was $67.42 \pm 5.6$ months. Decompression surgery with TLIF was applied to 67 patients, while decompression surgery without TLIF was applied to 95 patients. Overall,ASD developed in 40 patients (24.7\%). The type of stenosis was found to be a risk factor for ASD. Conclusion: Our results suggested that, although instrumentation and fusion applied to the surgical area caused an increase in stress and degeneration in the adjacent segment owing to immobilisation and stiffness in this area, the rate of increase did not rise with TLIF cage. Furthermore, the type of stenosis was determined to be a risk factor for ASD in our study.

Keywords: Adjacent segment, degenerative spine, fusion surgery
\end{abstract}

\section{INTRODUCTION}

Lumbar spinal stenosis (LSS) is a common clinical condition that is characterised by chronic lower back pain, radiculopathy and neurogenic claudication due to narrowing of the lumbar spinal central canal, lateral recess or foramen regions of the lumbar spine ${ }^{(1)}$. Lumbar decompression surgery has been indicated in patients with severe symptoms, and different surgical approaches have been previously described for this purpose ${ }^{(2)}$. In this context, lumbar decompression with posterior fusion has been demonstrated to be a valid and effective surgical approach ${ }^{(3)}$. Nevertheless, adjacent segment degeneration (ASD) is a challenging condition that is defined as degenerative changes occurring at the disk level adjacent to the operation site, comprising of disk/facet degeneration, instability and deformity ${ }^{(4)}$. Some supporting biomechanical and clinical data exists that suggest creation of a significant compensatory increase in the motion of the adjacent segment in spinal fusion as a result of increased rigidity of the fused segment. Consequently, the development of adjacent segment disease has been considered as a potential long-term complication after spinal fusion surgery ${ }^{(5)}$. ASD is considered as a cause of failed-back surgery; hence, the incidence and risk factors associated with ASD development warrant further investigation ${ }^{(4)}$.

Transforaminal lumbar interbody fusion (TLIF) has been recommended for patients undergoing decompression surgery and posterior instrumentation so as to provide circumferential arthrodesis and better stabilisation to decrease the risk of recurrence ${ }^{(5,6)}$. However, the available data are limited to the association between TLIF surgery and ASD in patients with posterior segmental instrumented $\operatorname{LSS}^{(7-10)}$. Therefore, the effect 
turkishspine

of TLIF cage applied in LSS decompression and fusion surgery on the development of ASD has been discussed in the present study.

\section{MATERIALS AND METHODS}

\section{Study Design and Participants}

This retrospective and comparative study was approved by the Adana City Training and Research Hospital Ethics Committee. (56-858/05.2020). We enrolled patients who underwent lumbar posterior segmental instrumentation and/or spinal decompression, without or with TLIF surgery for degenerative LSS in L4-5 or L5-S1 at our tertiary hospital during 2010-2015. In order to obtain a more homogeneous group, the L4-5 and L5S1 levels were included in the study. Patients who underwent revision surgery or dynamic stabilisation; had LSS due to disc herniation, cancer, inflammatory changes, lumbarisation or sacralisation; had significant spondylolisthesis or scoliosis and kyphosis; had incomplete data or did not attend follow-up examination visits and patients with both the types of stenosis (i.e. central and foraminal) were excluded. The patients were accordingly divided into two groups based on the status of ASD development. The clinical and demographic data of both the groups were comparatively evaluated.

\section{Surgical Procedure}

All patients were operated by the same surgical team (the same senior surgeon). All patients were preoperatively examined in detail with magnetic resonance imaging (MRI) and plane radiographs. The surgical approach in the prone position on the surgical table was applied to the groups of patients using posterior instrumentation (L4 to S1) with pedicle screws, total laminectomy, decompression and posterolateral fusion with iliac-crest auto-graft. In addition, discectomy and peek TLIF cage were applied to eligible patients. However, during the surgery, TLIF cage could not be applied to some of the patients due to certain incompatible situations (such as perioperatively deteriorated hemodynamic) or resistance to TLIF cage insertion (as a result of narrow gap of the disc that prevented cage insertion or insufficient imaging due to epidural bleeding). At this stage, auto-graft was placed in the anterior region of the TLIF cage as well as into the cage in the disc space. Then, compression was applied to ensure a tight attachment of the TLIF cage to the vertebral endplates. The procedure was completed by controlling all the patients with two planned (anteroposterior and lateral) fluoroscopy images.

\section{Evaluation of the ASD}

The diagnosis of ASD was based on MRI findings (preoperative and postoperative) with reference to the Pfirrmann classification of lumbar intervertebral disc degeneration ${ }^{(11)}$. According to this classification system, the comparative progress in the extent of degeneration at postoperative follow-up compared to that before the operation was accepted as ASD.

\section{Statistical Analysis}

SPSS software (SPSS 16 Inc., Chicago, IL, USA) was used for statistical analyses. The Kolmogorov-Smirnov test was applied to assess conformity of data to the normal distribution pattern. Descriptive data were expressed as mean \pm standard deviation or median (interquartile range) values. Categorical variables were compared using the chi-square test or Fisher's exact test. Student's t-test or Mann-Whitney U test was applied for comparisons between the groups. Binary logistic regression analysis was used to determine the associated risk factors. The presence of ASD was accepted as a dependent variable. $P<0.05$ was considered to be statistically significant.

\section{RESULTS}

A total of 162 patients (68 men, 94 women) of mean age $60.76 \pm 6.4$ years (age range: $37-89$ years) were evaluated. The mean follow-up period was established at $67.42 \pm 5.6$ months. Decompression surgery with TLIF was applied to 67 patients, while decompression surgery without TLIF was applied to 95 patients. Overall, ASD developed in 40 patients (24.7\%). Comparisons between patients without and with ASD is provided in Table 1. Central stenosis was found to be more common in ASD-positive patients.

The presence of ASD was determined to act as a dependent factor, while age ( $<65$ years and $\geqslant 65$ years), gender, body mass index [(BMI); $<25, \geqslant 25-30$ and $\left.\geqslant 30 \mathrm{~kg} / \mathrm{m}^{2}\right]$, type of stenosis (central or foraminal), TLIF application and the level of TLIF (L4-5 and L5-S1) acted as independent factors. The type of stenosis was found to be a risk factor for ASD. Central stenosis increased the risk for ASD by 2.7 times (Table 2).

\section{DISCUSSION}

The main goal of spinal fusion surgery is to maintain a solid arthrodesis of the spinal segments ${ }^{(12)}$. TLIF is a common surgical method administered along with decompression methods to offer several advantages, including preservation of the interspinous ligaments with minimal retraction of the dural sac, which causes less neurological injury and provides anterior support and $360^{\circ}$ fusion ${ }^{(13-15)}$. However, spinal fusion can induce ASD owing to biomechanical changes in the adjacent segment, such as increased movement and mechanical stress ${ }^{(5)}$. As mentioned in the literature, spinal fusion alone is ineffective in this condition ${ }^{(15)}$. The present finite element analysis indicated that decreased spinal lordosis may evoke overstress in the adjacent segment and predispose a patient to an increased risk of the pathological development of ASD. From this perspective, TLIF should be considered while planning spinal fusion procedures ${ }^{(15)}$. Due to ASD, clear symptoms requiring failed-back syndrome and revision surgery can be observed ${ }^{(16,17)}$. Previous studies have demonstrated reoperation rates due to ASD of $10-30 \%(16,17)$. As such, concerns about the pathophysiology and prevention of adjacent segment pathologies are indisputably 
Table 1. Comparison of patients with and without adjacent segment disease

\begin{tabular}{llll}
\hline Variables & $\begin{array}{l}\text { ASD }+ \\
(n=40)\end{array}$ & $\begin{array}{l}\text { ASD }- \\
(n=122)\end{array}$ & p value \\
\hline Age (years) & $62.12 \pm 4.0$ & $60.31 \pm 7.0$ & 0.090 \\
\hline Gender & $15(37.5)$ & $53(43.4)$ & 0.509 \\
\hline Male & $25(62.5)$ & $69(56.6)$ & 0.913 \\
\hline Female & $25.60 \pm 3.0$ & $25.53 \pm 3.4$ & 0.063 \\
\hline BMI (kg/m $\left.\mathbf{m}^{2}\right)$ & $67.73 \pm 3.8$ & $66.99 \pm 6.0$ & \\
\hline Follow-up (months) & & \\
\hline Type of stenosis & $11(27.5)$ & $59(48.4)$ & 0.021 \\
\hline Foraminal & $29(72.5)$ & $63(51.6)$ & - \\
\hline Central & $17(42.5)$ & $50(41.0)$ & 0.207 \\
\hline TLIF & $23(57.5)$ & $72(59.0)$ & \\
\hline Without-TLIF & & $75(61.5)$ & \\
\hline Level of TLIF & $29(72.5)$ & $47(38.5)$ & \\
\hline L4-5 & $11(27.5)$ &
\end{tabular}

Table 2. Regression analysis to determine the risk factors for the development of adjacent segment degeneration

\begin{tabular}{llllll}
\hline Variables & B & SE & Wald & Sig & Exp (B) \\
\hline Age & 0.446 & 0.453 & 0.971 & 0.325 & 1.562 \\
\hline Gender & 0.478 & 0.427 & 1.255 & 0.263 & 1.613 \\
\hline BMI & -0.035 & 0.321 & 0.012 & 0.913 & 0.966 \\
\hline Stenosis type & 1.006 & 0.424 & 5.638 & 0.018 & 2.734 \\
\hline TLIF & 0.100 & 0.402 & 0.061 & 0.804 & 1.105 \\
\hline TLIF Level & -0.637 & 0.439 & 2.102 & 0.147 & 0.529 \\
\hline
\end{tabular}

BMI: Body mass index, TLIF: Transforaminal interbody fusion, B: Unstandardized beta, SE: Standard error, Sig: Significance, Exp: Exponential

of great importance ${ }^{(12,18)}$. There is also a concern that posterior spinal fusion enhanced by intramuscular fusion can induce greater stiffness and present with potentially higher ASD rates $^{(16,17)}$. Although different surgical techniques have been compared in terms of ASD after degenerative lumbar diseases, the data on the association between TLIF and ASD development are scarce.

This study aimed to explore the association between TLIF and ASD development after decompression surgery on a selected group of patients with degenerative LSS only. Our findings revealed an overall frequency of ASD of $24.7 \%$ for this series of patients, which is consistent with previous clinical and biomechanical outcomes. ASD frequency was similar in patients who underwent TLIF surgery (25.4\%) in comparison with patients who did not (24.2\%). However, there is no consensus yet on the status of ASD developed or its relationship with the older age factor. While considering these points, it should be remembered that rigid and immobile areas created in the fusion area increases the stress and mobilisation on the adjacent segment. In a comprehensive meta-analysis, the occurrence of ASD after spinal fusion surgery was found to be consistent with a prevalence rate of $26 \%^{(7)}$. For instance, 94 studies with 34,716 patients from 19 countries were included in this study to reveal that the incidence of ASD on radiography was 4.8$92.2 \%$. In order to better analyse the development time of ASD, they performed subgroup analysis by ASD diagnosis time. In the 0.5 - to $\leqslant 2->2$ - to $\leqslant 5$ - and $>5$ - to $\leqslant 20$ - year periods, the respective radiograph ASD prevalence rates were $21.8 \%$ (16.027.6\%), 33.6\% (21.8-45.4\%) and 37.4\% (10.7-64.1\%). In another study of 112 patients with a mean age of 57 years (range: 15 85 years), the ASD rate with radiographic evidence of $20 \%$ was reported at a 2-year follow-up ${ }^{(8)}$. The number of studies need to be increased for the better understanding of age and follow-up time in this situation. Hilibrand and Robins ${ }^{(19)}$ and Levin et al.(20) argued that longer time is required for managing complications of ASD. In another study, although no significant difference was reported after a 1-year follow-up period, radiographic and clinical degeneration in the adjacent segments were detected in $43 \%$ and $24 \%$ of the patients after TLIF, respectively, after a minimum of 5 -year follow-up ${ }^{(9)}$, which is supported by some 
other studies ${ }^{(16)}$. Chen et al. ${ }^{(10)}$ investigated ASD after singlesegment posterior lumbar interbody fusion to report a $22 \%$ rate of ASD in patients with lumbar degenerative instability. In our study, no correlation was noted between the clinical outcomes and ASD after a single-level TLIF.

On the other hand, some previous studies have highlighted a significant association of ASD incidence with increased age ${ }^{(21,22)}$. Harrop et al.(23) systematically reviewed 27 articles and found that higher odds of radiographical ASD were associated with older patients. Cheh et al.(9), Yamashita et al.(24) and Sears et al. ${ }^{(25)}$ reported the age definition, which is a risk factor at the end of 5 -year follow-up, as $>50,>60$ and $>65$ years, respectively $(9,24,25)$. However, some other studies have reported no correlation between ASD incidence and age ${ }^{(26,27)}$. In fact, it has been argued that ASD is a normal degenerative process ${ }^{(17)}$. In this study, we did not detect any association between age and ASD.

Another factor that may contribute to ASD development is BMI. $A$ higher incidence of ASD in patients with $B M I \geqslant 25$ has been reported ${ }^{(28)}$. In contrast to the general literature, no significant difference was determined in this study between ASD-positive and ASD-negative groups, which can be attributed to the mean BMI of $<25 \mathrm{~kg} / \mathrm{m}^{2}$ of the patients in both the groups (ASDpositive and negative), which is also supported by some past studies $^{(29) .}$

The results of the present study also suggest that central stenosis is more common in ASD-positive patients and that central stenosis is a risk factor for ASD. There exists controversy about whether the level and number of fusion in lumbar degenerative diseases increase the incidence of ASD. In a study on the fusion level, spinal canal narrowing noted in the adjacent segment was considered as a risk factor for ASD following lumbar fusion surgery at a rate of $\geqslant 47 \%$. In this study, 3 - or 4-level fusions were reported to increase the risk of ASDrelated revision surgery by 3 -fold in comparison to single-level fusion ${ }^{(30)}$. In the present study, all patients showed single-level fusion, with no significant difference noted between the TLIF and non-TLIF fusion groups.

The main strength of the present study was that large and homogeneous patient groups with degenerative LSS alone facilitated better interpretation. However, the retrospective design of the present study and the difference in the surgical procedure between the two groups were the main study limitations. Nevertheless, the results of this study are noteworthy and can be considered to provide an insight into the mechanism of ASD after LSS surgery.

\section{CONCLUSION}

TLIF cage is used to generate fusion while performing decompression surgery in the degenerative spine. In the light of our study, although instrumentation and fusion applied to the surgical area can cause an increase in the level of stress and degeneration in the adjacent segment due to immobilisation and stiffness in this area, this rate does not increase with TLIF cage. In order to avoid revision due to implant failure and pseudo arthrosis, we believe that TLIF cage application does not have a negative effect when considering future ASD incidence. Further studies are recommended in prospective designs with larger patient series, including different levels of spinal stenosis.

\section{Ethics}

Ethics Committee Approval: This retrospective and comparative study was approved by the Adana City Training and Research Hospital Ethics Committee (56-858/05.2020).

Informed Consent: Retrospective study.

Peer-review: Internally peer-reviewed.

\section{Authorship Contributions}

Surgical and Medical Practices: E.K., F.S., Concept: E.K., F.S., Design: E.K., F.S., Data Collection or Processing: E.K., F.S., Analysis or Interpretation: E.K., F.S., Literature Search: E.K., F.S., Writing: E.K., F.S.

Conflict of Interest: No conflict of interest was declared by the authors.

Financial Disclosure: The authors declared that this study received no financial support.

\section{REFERENCES}

1. Wu L, Cruz R. Lumbar spinal stenosis. StatPearls [Internet]: StatPearls Publishing; 2018. Available at: https://www.ncbi.nlm.nih.gov/books/ NBK531493/

2. Yavin D, Casha S, Wiebe S, Feasby TE, Clark C, Isaacs A, et al. Lumbar fusion for degenerative disease: a systematic review and metaanalysis. Neurosurgery. 2017;80:701-15.

3. Lee $\mathrm{CH}$, Hyun SJ, Kim KJ, Jahng TA, Kim HJ. Decompression only versus fusion surgery for lumbar stenosis in elderly patients over 75 years old: which is reasonable? Neurol Med Chir (Tokyo). 2013;53:870-4.

4. Trivedi NN, Wilson SM, Puchi LA, Lebl DR. Evidence-based analysis of adjacent segment degeneration and disease after LIF: A Narrative Review. Global Spine J. 2018;8:95-102.

5. Wang H, Ma L, Yang D, Wang T, Liu S, Yang S, et al. Incidence and risk factors of adjacent segment disease following posterior decompression and instrumented fusion for degenerative lumbar disorders. Medicine (Baltimore). 2017;96:e6032.

6. Masevnin S, Ptashnikov D, Michaylov D, Meng H, Smekalenkov O, Zaborovskii N. Risk factors for adjacent segment disease development after lumbar fusion. Asian Spine J. 2015;9:239-44.

7. Xia XP, Chen HL, Cheng HB. Prevalence of adjacent segment degeneration after spine surgery: a systematic review and metaanalysis. Spine. 2013;38:597-608.

8. Poh SY, Yue WM, Chen LTJ, Guo CM, Yeo W, Tan SB. Two-year outcomes of transforaminal lumbar interbody fusion. J Orthop Surg. 2011;19:135-40.

9. Cheh G, Bridwell KH, Lenke LG, Buchowski JM, Daubs MD, Kim Y, et al. Adjacent segment disease followinglumbar/thoracolumbar fusion with pedicle screw instrumentation: a minimum 5-year follow-up. Spine (Phila Pa 1976). 2007;32:2253-7.

10. Chen BL, Wei FX, Ueyama K, Xie DH, Sannohe A, Liu SY. Adjacent segment degeneration after single-segment PLIF: the risk factor for degeneration and its impact on clinical outcomes. Eur Spine J. 2011;20:1946-50.

11. Urrutia J, Besa P, Campos M, Cikutovic P, Cabezon M, Molina M, et al. The Pfirmann classification of lumbar intervertebral disc 
degeneration: an independent inter-and intra-observer agreement assessment. Eur Spine J. 2016;25:2728-33.

12. Lan $T$, Hu SY, Zhang $Y-T$, Zheng $Y-C$, Zhang $R$, Shen $Z$, et al. Comparison between posterior lumbar interbody fusion and transforaminal lumbar interbody fusion for the treatment of lumbar degenerative diseases: a systematic review and meta-analysis. World Neurosurg. 2018;112:86-93.

13. Asil K, Yaldiz CJM. Retrospective comparison of radiological and clinical outcomes of PLIF and TLIF techniques in patients who underwent lumbar spinal posterior stabilization. Medicine (Baltimore) 2016;95:e3235.

14. Talia AJ, Wong ML, Lau HC, Kaye AH. Comparison of the different surgical approaches for lumbar interbody fusion. J Clin Neurosci. 2015;22:243-51.

15. Cheng X, Zhang K, Sun X, Zhao C, Li H, Ni B, et al. Clinical and radiographic outcomes of bilateral decompression via a unilateral approach with transforaminal lumbar interbody fusion for degenerative lumbar spondylolisthesis with stenosis. Spine J. 2017; 17:1127-33.

16. Maragkos GA, Motiei-Langroudi R, Filippidis AS, Glazer PA, Papavassiliou E. Factors predictive of adjacent segment disease after lumbar spinal fusion. World Neurosurg. 2020;133:e690-4.

17. Burch MB, Wiegers NW, Patil S, Nourbakhsh A. Incidence and risk factors of reoperation in patients with adjacent segment disease: $A$ meta-analysis. J Craniovertebr Junction Spine. 2020;11:9-16.

18. Zhao X, Du L, Xie Y, Zhao J. Effect of lumbar lordosis on the adjacent segment in transforaminal lumbar interbody fusion: a finite element analysis. World Neurosurg. 2018;114:e114-20.

19. Hilibrand AS, Robbins M. Adjacent segment degeneration and adjacent segment disease: the consequences of spinal fusion? The Spine Journal. 2004;4:S190-S4.

20. Levin DA, Hale JJ, Bendo JA. Adjacent segment degeneration following spinal fusion for degenerative disc disease. Bulletin of the NYU hospital for joint diseases. Bull NYU Hosp Jt Dis. 2007;65:29-36.
21. Yee TJ, Terman SW, La Marca F, Park PJJoCN. Comparison of adjacent segment disease after minimally invasive or open transforaminal lumbar interbody fusion. J Clin Neurosci. 2014;21:1796-801.

22. Radcliff KE, Kepler CK, Jakoi A, Sidhu GS, Rihn J, Vaccaro AR, et al. Adjacent segment disease in the lumbar spine following different treatment interventions. 2013;13:1339-49.

23. Harrop JS, Youssef JA, Maltenfort M, Vorwald P, Jabbour P, Bono $\mathrm{CM}$, et al. Lumbar adjacent segment degeneration and disease after arthrodesis and total disc arthroplasty. Spine (Phila Pa 1976). 2008;33:1701-7.

24. Yamashita K, Ohzono K, Hiroshima K. Five-year outcomes of surgical treatment for degenerative lumbar spinal stenosis: a prospective observational study of symptom severity at standard intervals after surgery. Spine (Phila Pa 1976). 2006;31:1484-90.

25. Sears WR, Sergides IG, Kazemi N, Smith M, White G], Osburg B. Incidence and prevalence of surgery at segments adjacent to a previous posterior lumbar arthrodesis. Spine J. 2011;11:11-20.

26. Aiki H, Ohwada O, Kobayashi H, Hayakawa M, Kawaguchi S, Takebayashi T, et al. Adjacent segment stenosis after lumbar fusion requiring second operation. J Orthop Sci. 2005;10:490-5.

27. Lee CS, Hwang CJ, Lee SW, Ahn YJ, Kim YT, Dong HL, et al. Risk factors for adjacent segment disease after lumbar fusion. Eur Spine J. 2009;18:1637-43.

28. Hashimoto K, Aizawa T, Kanno H, Itoi E. Adjacent segment degeneration after fusion spinal surgery-a systematic review. Int Orthop. 2019;43:987-93.

29. Min JH, Jang JS, Jung BJ, Lee HY, Choi WC, Shim CS, et al. The clinical characteristics and risk factors for the adjacent segment degeneration in instrumented lumbar fusion. J Spinal Disord Tech. 2008;21:305-9.

30. Yugué I, Okada S, Masuda M, Ueta T, Maeda T, Shiba K. Risk factors for adjacent segment pathology requiring additional surgery after singlelevel spinal fusion: impact of pre-existing spinal stenosis demonstrated by preoperative myelography. Eur Spine J. 2016;25:1542-9. 